\title{
Optimization of hydraulic modes of distribution heat networks by dynamic programming*
}

\author{
Alexandr V. Lucenko ${ }^{1, \dagger}$ \\ ${ }^{1}$ Melentiev Energy Systems Institute of Siberian Branch of the Russian Academy of Sciences (ESI \\ SB RAS), Pipeline Energy Systems Department, 130, Lermontov str., Irkutsk, Russia, 664033
}

\begin{abstract}
An original modification of the dynamic programming method is proposed, which is designed to optimize the hydraulic modes of distribution heat networks, based on special properties of the problem. It is demonstrated that the proposed modification of the dynamic programming method has a high computational efficiency in comparison with possible alternative methods of discrete-continuous optimization.
\end{abstract}

\section{Introduction}

Russia raises the questions of energy efficiency, and heat supply systems (HSS) have large energy-saving resources due to the non-optimal nature of their operating modes. The task of planning the operating modes of the HSS arises in preparation for the heating season. In practice, this problem is solved by multivariate calculations of the mode [1,2,Ошибка! Источник ссылки не найден.]. The choice of ways to organize the modes is assigned to the engineer, and the quality and optimality of decisions depends both on his experience and qualification, and on the scale and complexity of the HSS. This does not guarantee the optimality, and often the acceptability, of the developed regimes. This increases the relevance of the development and application of methods and software packages for calculating the optimal HSS. There are some factors, hindering the automation of solving this problem. These are: high dimensionality of the applied flow distribution models, their nonlinearity, several optimality criteria, discreteness of variables, etc. For these reasons, there are no methods and software packages that are suitable for practical use.

Recently there are works devoted to this problem. Most of them are devoted to TSS of small dimension, for example [4]. Genetic algorithms are used in [5], which is associated with large computational costs. Often, works devoted to the optimization of TSS regimes are of a private nature and limited scope. Thus, in [6], the optimization of the mode is performed in order to minimize the total fuel consumption, and in [7] - the total power consumed at pumping stations. Real-time management task is given enough attention, for example $[6,8,9]$. The tasks of reconstruction and development are devoted to a large number of works $[10,11]$. In all these articles, in order to overcome the dimensionality

\footnotetext{
${ }^{*}$ The research was carried out within the project III.17.4.3 of the Fundamental research program of SB RAS (AAAA-A17-117030310437-4) with finance support of RFBR and the Government of Irkutsk Region in the framework of research project № 17-48-380021

†Corresponding author: luc_alex@mail.ru
} 
problem, the HSS calculation schemes are aggregated. This can adversely affect the accuracy and optimality of solutions.

To overcome the dimension problem ISEM SB RAS developed a multi-level approach to optimizing HSS modes [12]. It includes the following steps:1) decomposition of TSS into backbone (BHN) and distribution (DHN) heat networks; 2) finding the limits of the mode parameters at decomposition points that guarantee the existence of permissible modes of DHN; 3) optimization of the BHN mode, taking into account these limitations; 4) optimization of DHN modes taking into account the results of the previous stage. At the BHN level, a part of the heat networks, which contains all heat sources and pumping stations, is looped (in a single-line image), passive branched networks to the end users - to the DHN level. This allows you to take into account the costs associated with maintaining the mode, only when optimizing the modes of BHN [13].

In this paper, we consider the problem related to item 4 of this approach and the adaptation of the method of dynamic programming (DP) to its solution. This task is important in the development of modes for a single DHN, or HSS, which has a single source and branched passive thermal networks.

At the moment there are many implementations of the DP method used to optimize the modes and parameters of the pipeline systems tree structure [10]. For the case of tree-like pipeline systems, the conditions for the applicability of the DP are fulfilled: the additivity of the objective function and Bellman's optimality principle [14]. Sumarokov S.V. for the general case of multi-loop networks with an unknown flow distribution proposed a method of multi-loop optimization (MLO) [15], that assumes the iterative process. The main problem of applying DP to multi-circuit pipeline systems with known flow distribution is related to the need to comply with Kirchhoff's second law on contours. For this case, a modification of the MLO method [16], which assumes the iteration process, at each iteration, the pressure calculations are carried out by the DP method on a tree-like form of the calculated scheme with subsequent correction of pressure limitations. The main drawback of this approach is its iterativity and poor convergence.

\section{Problem statemant}

The main requirements for DHN modes are related to their permissibility and minimum labor input for their provision. The last can be reduced to minimizing the number of controls. It is advisable to reduce the overall level of pressure. This makes the task of the task of two-criteria optimization.

The main elements of the DHN are pipelines and consumers. We denote $I_{P L}$ and $I_{C}$ - sets of indexes of branches modeling elements of the first and second types so that $I_{P L} \cap I_{C}=\varnothing$ and $I_{P L} \cup I_{C}=I-$ set of indices of all branches of DHN. We will use the controlled flow distribution model [16]:

$$
\mathrm{U}(\mathbf{X})=\left(\begin{array}{c}
\mathbf{U}_{1}(\mathbf{x}, \mathbf{Q}) \\
\mathbf{U}_{2}(\mathbf{P}, \mathbf{y}) \\
\mathbf{U}_{3}(\mathbf{x}, \mathbf{y}, \mathbf{z})
\end{array}\right)=\left(\begin{array}{c}
\mathrm{A} \mathbf{x}-\mathbf{Q} \\
\mathrm{A}^{\mathrm{T}} \mathbf{P}-\mathbf{y} \\
\mathbf{y}-\mathbf{f}(\mathbf{x}, \mathbf{z})
\end{array}\right)=\mathbf{0},
$$

where $A$ - incidence $(m \times n)$-matrix of a connected oriented graph of the calculated scheme of the HSS; $n, m$ - number of branches and nodes of the calculated scheme; $\mathbf{Q}-m$ dimensional vector of nodal flows; $\mathbf{P}-m$-dimensional vector of nodal pressures; $\mathbf{x}, \mathbf{y}-n$ dimensional vectors of flow and pressure differentials on the branches of the hydraulic circuit; $\mathbf{f}(\mathbf{x}, \mathbf{z})-n$-dimensional vector function with elements $f_{i}\left(x_{i}, z_{i}\right)=z_{i} s_{i} x_{i}\left|x_{i}\right|$ [16], $i=\overline{1, n} ; \mathbf{z}-$ control vector; $s_{i}$ - rated hydraulic resistance; $\mathbf{X}=\{\mathbf{R}, \mathbf{z}\}-$ vector of real variables; $\mathbf{R}=\{\mathbf{P}, \mathbf{x}, \mathbf{y}, \mathbf{Q}\}$ - vector of mode parameters. 
As the boundary conditions for the DHN, pressures are set in the nodes of the connection between the DHN and the BHN (in the supply and return pipelines) and the flows in all other nodes. Technical and technological requirements for the permissibility and feasibility of the mode and technologically permissible limits for changing controls are reduced to the need to ensure conditions $\underline{\mathbf{X}} \leq \mathbf{X} \leq \overline{\mathbf{X}}$, where $\overline{\mathbf{X}}, \underline{\mathbf{X}}$ - The upper and lower constraints on the variation of the $\mathbf{X}$ components. We introduce the vector of Boolean variables $\boldsymbol{\delta}$, whose components denote the presence of control on the i-th branch of the design scheme $\left(i \in I_{P L}\right)$, and inequality $\underline{z}_{i} \leq z_{i} \leq \underline{z}_{i}+\delta_{i}\left(\bar{z}_{i}-\underline{z}_{i}\right)$. Then the system of inequalities turns into

$$
\underline{\mathbf{X}} \leq \mathbf{X} \leq \overline{\mathbf{X}}(\boldsymbol{\delta}) .
$$

As an optimality criterion for the number of controls, we use $F_{z}=\sum_{i \in I_{P L}} \delta_{i}$ [16]. let $F_{P}=\sum_{j \in J} P_{j} / m$ [16] be an indicator of the overall level of pressure in the network. Proceeding from practice, the task of two-criteria optimization with discrete main criterion is most expedient. We apply the principle of lexicographic ordering of criteria. Then the problem of two-criteria optimization will take the form

$$
\min F_{P} \text { under constraints (1), (2) and } F_{z} \leq F_{z}^{*} \text {. }
$$

Here $F_{z}^{*}$ - the least number of controls on the passive branches necessary to achieve an permissible mode. Known: the topology of the calculated scheme (matrix $A$ ), boundary conditions, the coefficients of the hydraulic characteristics of the branches $\left(s_{i}\right)$, the limits of the permissible change of the unknowns. $(\underline{\mathbf{X}}, \overline{\mathbf{X}})$.

\section{Modified DP method}

Features of DHN are: 1) tree in a single-line representation and multi-contour in a two-line; 2) the supply and return pipelines are symmetrical to each other and connected through consumers whose coolant flow rates are fixed; 3) fixed flow distribution. Due to these features, the DHN calculated scheme can be reduced to one branch using techniques of equivalent of consecutively and in parallel connected branches.

To facilitate control over the implementation of Kirchhoff's second law on contours, it is proposed to "reduce" the contours to a parallel connection of the two branches. To preserve the ability to apply DP on "reduced" contours, techniques for the equivalence of piezometric graphs of consecutively and parallel connection of branches are proposed. The direct approach of the proposed approach is to reduce the piezometric graphs of the DHN calculated scheme with the rejection of unacceptable and non-optimal variants, leaving only the optimal piezometric graph. The reverse approach consists in its restoration over all elements of the original DHN calculated scheme.

We introduce the set of indices of all nodes $J$ and the set of branches of the calculated scheme $L$. Let all the branches $\left(L_{i}, i \in I\right)$ be directed along the flow. Denote the initial and final nodes of the branch as $f_{i}$ and $l_{i}$. The intervals of pressure change in the nodes will be divided into pockets $\left(P_{j}^{r}\right), j \in J$. We assign to each branch a set of segments of piezometric graphs ( $\left.L_{i}=\bigcup\left\{g_{i}^{k}\right\}\right)$ starting at the initial node of the branch, and terminating in the final one. To each such segment we assign the value of the increment of the optimality criterion $\left(\Delta F_{i}^{k}\right)$. We denote the initial pocket as $\phi_{i}^{k}$, final $-\varphi_{i}^{k}$. 
The technique of equating parallel branches of one. If on two branches connected in parallel $(i 1, i 2)$, there is a pair of segments of piezometric graphs for which $\phi_{i 1}^{k 1}=\phi_{i 2}^{k 2}$ и $\varphi_{i 1}^{k 1}=\varphi_{i 2}^{k 2}$, then such a pair becomes a segment of the piezometric graph of the equivalent branch beginning and ending in the corresponding pockets. The values of the increments of the optimality criterion are added together. The segments of the piezometric graphs, not included in any pair, are rejected.

When equating two branches $(i 1, i 2)$ connected in series $\left(f_{i 2}=l_{i 1}\right)$, it is proposed to find all such pairs of segments of piezometric graphs on these branches $\left(g_{i 1}^{k 1}, g_{i 2}^{k 2}\right)$ that the node $f_{i 2}$ satisfies the relation $\varphi_{i 1}^{k 1}=\phi_{i 2}^{k 2}$. Of the several pairs of segments connecting the same pockets, the best remains. In the case of solving a multicriteria problem with lexicographical ordering of criteria, for example (3), after rejecting by the main criterion, it is proposed to reject pairs by secondary criteria. Each of the pairs found is transformed into a section of the piezometric graph with the corresponding initial and final pockets. The increments in the optimality criterion are added together.

To restore the optimal trajectory of the phase variables, it is necessary for each equivalent branch to remember which fragment it is equivalent to, and for each equivalent path of the phase variable, which segments it equals.

Unlike the MLO method, the proposed method does not imply iterative processes. The advantage of the proposed method over methods based on the method of internal points [16] is the linear growth in DHN dimension of the necessary computational costs.

\section{Computational experiments}

To test the efficiency of the proposed method, a series of computational experiments was conducted. Here is a typical example. For a test RTS, the scheme of which in a single-line image is shown in Fig. 1-a, the problem (3) was solved. In Fig. 1-b shows its calculated scheme. Branches 8 to 11 are consumers. Coolant flow rates are set on them $x_{8}=100, x_{9}=50$, $x_{10}=100, x_{11}=150$. The minimum pressure drop across these branches is 15 . The fluxes in nodes 2 to 15 are zero. $P_{1}=100$ water meter, $P_{16}=30$. The pressure in node 9 was limited from below by a value of 70 water meter, in node 6 - from above with a value of 60 . The resistance vector is $(0,00003125,0,0005,0,00011111,0,0005,0,0004,0,0003,0,0002222$, $0,0001,0,0001,0,0001,0,0001,0,0005,0,0004,0,0003,0,0002222,0,0005,0,00011111$, $0,00003125)$.

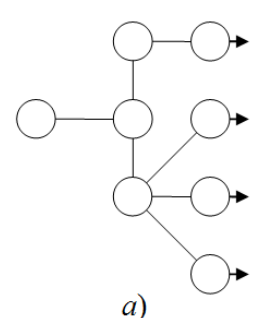

a)

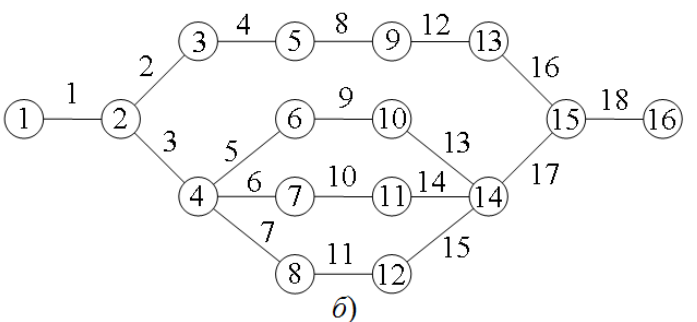

Fig. 1. Scheme of test DHN.

To achieve the permissibility of the regime, it was necessary to install throttling devices on branches 5 and 12 . The pressure vector found is equal to $(100,95,90,85,85,60,82,80$, $70,45,47,79,40,44,35,30)$ water meter. The verification of the results was carried out by the majorizing sequence method [16]. The verification showed the correctness of the results 
of the proposed method. Also, the running time of the proposed method was significantly shorter than the running time of the majorizing sequence method.

Computational experiments showed the efficiency of the proposed method.

\section{Conclusions}

1. The mathematical formulation of the discrete-continuous problem of two-criteria optimization of the DHN hydraulic regimes arising at the stage of regime planning is given. Currently, there are no special methods for its solution, suitable for practical use.

2. To solve this problem, an original method is proposed, consisting in folding with the rejection of unrealizable and suboptimal piezometric graphs of the DHN calculation scheme into a piezometric graph of one branch with its subsequent unfolding on the entire DHN.

3.It is demonstrated that the proposed method allows solving multicriterion optimization problems.

4. The proposed method is implemented in the form of a research program. The results of its application for numerical calculations illustrating its effectiveness are presented.

\section{References}

1. H. Boysen, J.E. Thorsen, EHP 4 (2007)

2. F. Wernstedt, P. Davidsson, Proc. of the First Intern. Conf. on Agreement Technologies, Dubrovnik, Croatia, October 15-16, 2012, p. 423-437. (2012)

3. N.N. Novitsky, M.G. Sukharev and others, Pipeline energy systems. Methodical and applied problems of mathematical modeling. (2015)

4. D. Buoro, P. Pinamonti, M. Reini, App. Energy, 124, (2014)

5. A. Sciacovelli, E. Guelpa, V. Verda, Proc. of Intern. Mechanical Engineering Congr. IMECE2013: 2013 ASME \& Exposition San Diego, California, November 15-21.

6. X.S. Jiang, Z.X. Jing, Y.Z. Li, Q.H. Wu, W.H. Tang, Energy, 64, (2014.)

7. S. Cosentino, E. Guelpa, R. Melli, A. Sciacovelli, E. Sciubba, C. Toro, V. Verda, Proc. of the Intern. Mechanical Engineering Congr. And Exposition «ASME» 2014 November 14-20. Montreal, Quebec, Canada. (2014)

8. Z.X. Jing, X.S. Jiang, Q.H. Wu, W.H. Tang, B. Hua, Energy, 73, (2014.)

9. T. Laajalehto, M. Kuosa, T. Makila, M. Lampinen, R. Lahdelma, Appl. Therm. Eng. 69, (2014)

10. A.P. Merenkov, V.Y. Hasilev, Theory of hydraulic circuits. (1985)

11. C. Haikarainen, F. Pettersson, H. Saxen, Chem. eng. trans. 35, (2013)

12. A.V. Lucenko, N.N. Novitsky, Methodological issues of reliability research for large energy systems. Issue. 64. (2014)

13. A.V. Lucenko, N.N. Novitsky, J. Global Optim., 66, 1 (2016)

14. R.E. Bellman, Dynamic Programming. (1957).

15. S.V. Sumarokov, A.V. Hramov, Methods of optim. oper. research (appl. math.). (1976)

16. A.P. Merenkov, E.V. Sennova, S.V. Sumarokov, et al. Mathematical modeling and optimization of heat, water, oil and gas supply systems. (1992)

17. A.V. Lutsenko, N.N. Novitsky, Sci. Bull. NSTU. 64, 3. (2016) 